\section{BACKGROUND}

Quality of life (QoL) has been variously reported as normal or impaired in adults with congenital adrenal hyperplasia $(\mathrm{CAH})$. To explore the reasons for this discrepancy we investigated the relationship between QoL and other health

\section{METHODS}

\section{Patient recruitment}

The CaHASE cohort is a cross-sectional study of CAH adults recruited from 17 specialized British Endocrine centres. The study protocol was approved by West Midlands (MREC/03/7/086) and registered with ClinicalTrials.gov (NCT00749593).

All centres contacted adult patients (18 years or older) with a confirmed diagnosis of CAH currently under their care. Recruitment started August 2004 and ended July 2007. All participants gave written informed consent

\section{Outcome measures}

QoL: SF-36 questionnaires.

Androstenedione, 17-OHP, testosterone.

Waist circumference, triglycerides and HDL cholesterol, glucose and insulin (HOMA-IR).

Systolic and diastolic BP, gene mutations.

Type (hydrocortisone, prednisolone and dexamethasone) and dose of glucocorticoid expressed as prednisolone dose equivalent (PreDEq).

\section{Biochemistry measurements}

Performed at local laboratories (all participate in the UK NEQAS scheme for quality control of steroid immunoassays) Serum insulin was measured centrally using an ultrasensitive enzyme-linked immunosorbent assay (DRG instruments, Marburg, Germany).

Mutation analysis, mutation groups and in vitro analysis of CYP21A2 mutations

CYP21A2 gene deletion and chimeric genes were detected using a commercially available multiplex-ligation probe amplification (MLPA) strategy following the manufacturers protocol (mrc-Holland, Amsterdam, The Netherlands). Pseudogenederived CYP21A2 point mutations were performed by targeted multiplex-mini sequencing after allele specific PCR amplification of the CYP21A2 gene. If no common mutations were detected, direct DNA sequencing of the entire CYP21A2 gene was performed.

Patients were categorized into established CYP21A2 mutation groups according to their genotype with the less severe mutation determining the group: Null (mutations absent in vitro activity), A (intron 2 splice site mutation), B (mutations such as the $1172 \mathrm{~N}$ mutation and mutations with $1-10 \%$ in vitro residual enzyme activity), C (mutations such as P30L, V281L, and P453S or above $20-30 \%$ in vitro 21 -hydroxylase activity).

\section{Data analysis Cross-sectional analysis of 151 adults with 21-} hydroxylase deficiency (50M: 47 with classic and 3 with non-classic $\mathrm{CAH}$; 101F: 75 with classic and 26 with non-classic $\mathrm{CAH}$ ) aged 1869 years in whom QoL (SF-36). Glucocorticoid regimen, anthropometric and metabolic measures were recorded. QoL was expressed as z-scores calculated from matched controls (14,430 subjects from UK population, courtesy of Professor John Brazier, Sheffield University). Relationships were examined between QoL and outcome measures. Principal Components Analysis (PCA) was undertaken to identify clusters of associated clinical and biochemical features and the principal component (PC) scores used in regression analysis as predictor of QoL.

\section{RESULTS}

QoL scores were associated with type of glucocorticoid treatment for vitality $(P=0.002)$ and mental health $(P=0.011)$, with higher z-scores indicating better $Q \circ L$ in patients on hydrocortisone than in patients receiving prednisolone or dexamethasone $(P<0.05)$. QoL did not relate to PreDEq or mutation severity. PCA identified three PCs (PC1, disease control; $\mathrm{PC} 2$, adiposity and insulin resistance; $\mathrm{PC} 3$, blood pressure and mutations) that explained $61 \%$ of the variance in observed variables. Stepwise multiple regression analysis demonstrated that PC2 (comprising waist circumference, serum triglycerides, HOMA-IR and HDL-cholesterol) was associated with QoL scores, specifically impaired physical functioning, bodily pain, general health, Physical Component Summary Score $(P<0.001)$ and vitality $(P=0.002)$.

Table 1. Quality of life SF-36 standardized scores and z-scores for CAH adults, based on reference data obtained from Professor John Brazier (University of Sheffield, UK) constructed a representative random sample of 14,430 subjects from the UK population aged 18 to 79 years. For every CAH patient, twenty sex- and age-matched controls were randomly selected from the representative reference samples and $z$-scores generated. Adjustment for age and sex was performed by transformation of all domain score values from patients into age- (decade) and sex-adjusted z-scores. Red $=$ Physical health domains; Blue $=$ Mental health domains .

\begin{tabular}{|l|c|c|}
\hline & Standardized scores & Z-scores \\
\hline Physical function & Mean \pm SD & Mean \pm SD \\
\hline Role limitations due to physical problems & $81.2 \pm 25.2$ & $-0.74 \pm 1.53$ \\
\hline Bodily pain & $74.2 \pm 37.7$ & $-0.71 \pm 1.33$ \\
\hline General health & $68.4 \pm 27.8$ & $-0.45 \pm 1.26$ \\
\hline Vitality & $56.1 \pm 25.4$ & $-0.61 \pm 1.09$ \\
\hline Social functioning & $50.4 \pm 21.6$ & $-0.87 \pm 1.21$ \\
\hline Role limitations due to emotional problems & $73.2 \pm 26.9$ & $-0.54 \pm 1.02$ \\
\hline Mental health & $69.3 \pm 40.1$ & $-0.91 \pm 1.66$ \\
\hline
\end{tabular}

Table 2. Regression coefficients $(\beta)$ and explained variances obtained from stepwise multiple regression analysis using the three principal components (PC1, PC2 and PC3) as predictor variables and health related QoL SF-36 questionnaire, age- and sex-adjusted, $z$-scores as dependent variables. Only $\mathrm{PC2}$, reflecting adiposity and insulin resistance (waist circumference, serum triglycerides, HOMA-IR and HDL-cholesterol) was retained showing adverse relationships with physical function, bodily pain, general health, vitality and Physical Component Summary Score. Additional adjustment for PreDEq, or type of glucocorticoid replacement (hydrocortisone, prednisolone and dexamethasone) did not change these relationships. Red $=$ Physical health domains; Blue $=$ Mental health domains

\title{
Predictor variable
}

Dependent variables

Physical function

Role limitations due to physical problems

Bodily pain

General health

Physical Component Summary Score

Vitality

Social functioning

Role limitations due to emotional problems

Mental health

Mental Component Summary Score

\begin{tabular}{|c|c|c|}
\multicolumn{3}{|c|}{ (PC2: Adiposity and insulin resistance) } \\
\hline$\beta(\mathbf{9 5 \%} \mathrm{Cl})$ & $\boldsymbol{P}$ & $\boldsymbol{r}^{2}(\mathbf{\%})$ \\
\hline$-0.72(-1.11$ to -0.35$)$ & $<0.001$ & 19.9 \\
\hline Not significant & --- & --- \\
\hline$-0.55(-0.82$ to -0.28$)$ & $<0.001$ & 21.6 \\
\hline$-0.50(-0.80$ to -0.20$)$ & 0.001 & 16.0 \\
\hline$-0.58(-0.83$ to -0.33$)$ & $<0.001$ & 26.4 \\
\hline$-0.40(-0.65$ to -0.16$)$ & $\mathbf{0 . 0 0 2}$ & $\mathbf{1 5 . 5}$ \\
\hline Not significant & --- & --- \\
\hline Not significant & --- & --- \\
\hline Not significant & --- & --- \\
\hline Not significant & --- & --- \\
\hline
\end{tabular}

Influence of individual components of the adiposity and insulin resistance on physical functioning (Fig. 1) and on vitality QoL (Fig. 2): Error plots with mean and 95\% confidence limits showing the size of waist circumference (A), levels of triglycerides (B) and HDL cholesterol (C), and HOMA-IR (D) according to the physical functioning (Fig. 1) or vitality (Fig. 2) domain categorized into three groups based on z-scores $\geq 0(n=69),<0$ to $-1(n=36)$ and $<-1(n=46)$. Post hoc analysis: ${ }^{*} P<0.05,{ }^{* *} P<0.01,{ }^{* * *} P<0.001$ compared to group with $\mathrm{z}$-score $\geq 0$.

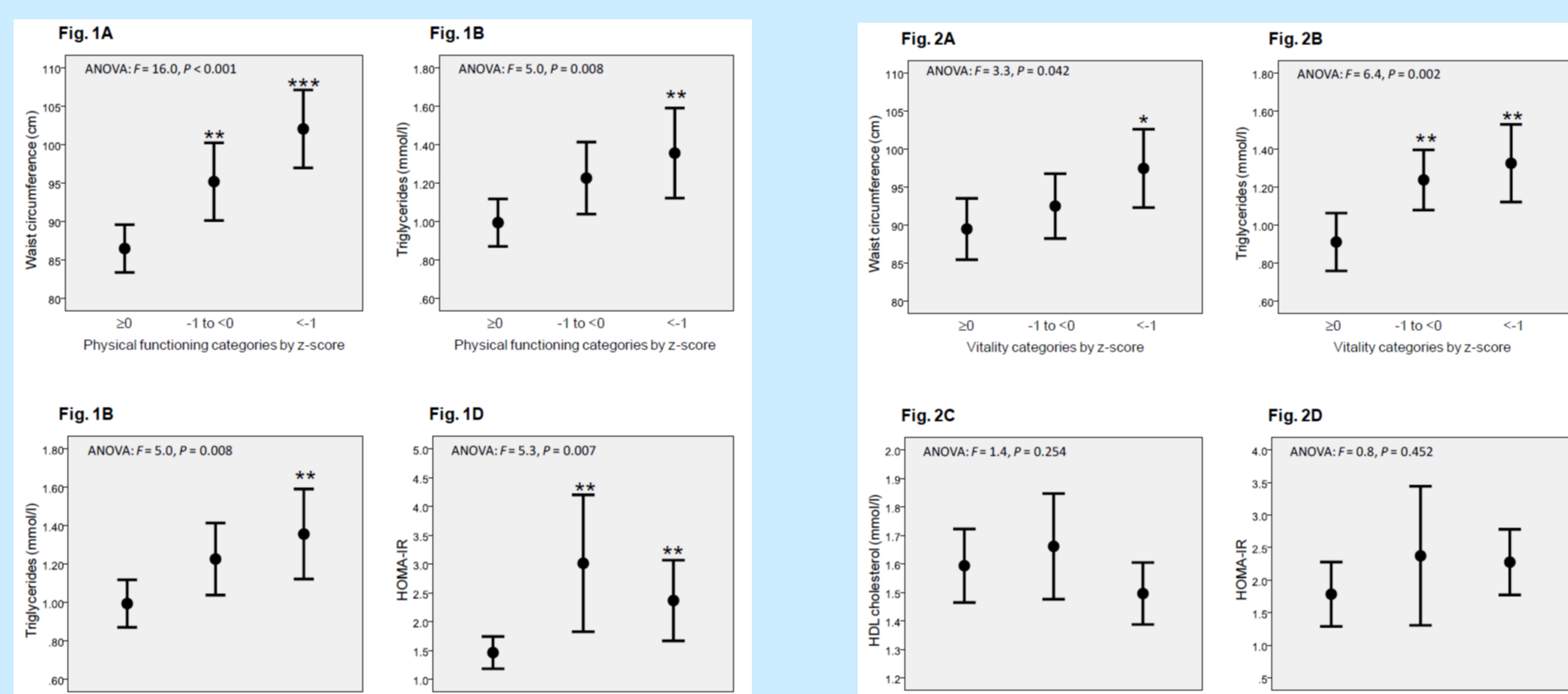

\section{SUMMARY OF FINDINGS IN ADULT CAH PATIENTS}

- QoL was impaired in this cohort of patients.

- Increased adiposity and Insulin resistance were associated with impaired QoL, predominantly in the physical health domains.

CONCLUSIONS

Increased adiposity and insulin resistance are associated with impaired QoL in adults with CAH. Further studies are justified to establish whether optimising the choice of glucocorticoid treatment and/or weight loss can improve QoL in this disadvantaged patient group.
ACKNOWLEDGEMENTS AND DECLARATION OF INTEREST project and The Clinical Endocrinology Trust for financial support. Professor Richard Ross is a founding director of Diurnal Ltd. 